\title{
Natural Circulation in a Pressurized Water Reactor with a Combined Single and Two-Phase Mode
}

\author{
Mohammed W. Abdulrahman' ${ }^{1}$, Mikdam M. Saleh ${ }^{2}$, Jonathan Anand ${ }^{3}$ \\ ${ }^{1}$ Rochester Institute of Technology \\ Dubai, UAE \\ mwacad@rit.edu; mikdam2@yahoo.com \\ ${ }^{2}$ University of Baghdad \\ Baghdad, Iraq \\ ja3127@rit.edu
}

\begin{abstract}
In this paper, a theoretical model is used to analyse the natural circulation phenomena relevant to small breaks for PWRs. The model utilizes the one-dimensional approach and the quasi-steady hypothesis and solves analytically the loop momentum balance together with the conservation of mass and energy, and develops expressions for the core flow rate, core inlet and outlet temperature, and core temperature difference as a function of primary pressure. The model should be capable of analysing the combined single and twophase mode of natural circulation during a small break LOCA without High Pressure Emergency Core Cooling (ECC) Injection. Also, the model is used to investigate the effect of core power on the analytical solution. The results of this paper are compared with a previous experimental result and show a reasonable agreement.
\end{abstract}

Keywords: Natural circulation; PWR; LOCA; Single phase; Two phase

\section{Introduction}

In PWRs, natural circulation is considered as an essential mechanism to reject the decay heat of the reactor core during a loss of coolant accident. Accidents can occur in a number of ways, i.e., pump seal failure, steam generator tube failure, or safety valves sticking open, and instrument-tube failure, in addition to simple pipe breaks or leaks. It is noted that any new nuclear power plant must run a natural circulation test as one of the requirements for an operating license. In the natural circulation in PWRs, the primary coolant flows by a gravitational driving force in a closed primary loop. The gravitational driving force is resulted from the coolant density differences in the primary loop, where the density is low in the reactor core (heating source) and high in the steam generators (heat sink). To create the gravitational driving force, the heat source must be below the heat sink. There are four modes of NC in PWRs, which are; single-phase, combined single and two-phase, twophase, and reflux condensation. In this paper, combined single and two-phase flow of NC is investigated.

Natural circulation has been studied by many researches. Zvirin presented a survey on the theoretical and experimental work on single-phase natural circulation loops (thermosyphon). It included modelling methods to describe steady-state flows, transients and stability characteristics of the various loops [1]. Ward and Shimeck and Johnsen showed that the physics of natural circulation process is a manometric momentum balance between the hydrostatic head in the down-comer driving the core inlet flow, and the pressure losses incurred in venting the resulting two-phase flow from the core outlet to the heat exchanger [2, 3]. Fernandez has studied some natural circulation loops that involve two-phase flows (e. g., thermosyphon reboilers and natural circulation reflux boiling in LWR loops) [4]. Abdulrahman has modelled analytically the steady-state heat transfer behaviour of a fluid flowing through a packed bed and determined the temperature distributions and heat transfer rates $[5,6]$.

In this paper, a theoretical analysis is developed to investigate the $\mathrm{NC}$ for combined single and two-phase flow in a PWR during a small break LOCA. Starting from a single-phase natural circulation condition at blow-down initiation, the downcomer flow remains at about the initial value. As more coolant leaving the system via the break, combined single and twophase natural circulation is induced. During this mode of NC, saturated conditions and bubble formation throughout the core and hot leg are produced, while sub-cooled water is produced throughout the down-comer and the entrance of the core. 


\section{Steady State One-Dimensional Natural Circulation Flow}

For a flow in a one-dimensional positive $s$-direction, the steady state equations of continuity, momentum, and energy are respectively [7];

$$
\begin{gathered}
\frac{d u}{d s}=-\frac{u}{\rho} \frac{d \rho}{d s} \\
\rho u \frac{d u}{d s}=-\frac{d P}{d s}-F_{s}-\rho g \cos (\theta) \\
\rho A u \frac{d h}{d s}=-\frac{d q}{d s}
\end{gathered}
$$

where $u$ is the flow velocity, $\rho$ is the fluid density, $P$ is the primary pressure, $g$ is the gravitational acceleration, $A$ is the cross sectional area of the flow channel, $h$ is the specific enthalpy, and $q$ is the heat added to (or rejected from) the coolant. In terms of the mass flow rate $W$, the flow velocity $u$ can be expressed as; $u=\frac{W}{\rho A}$, where $\rho$ is the fluid density and $A$ is the flow cross sectional area. For turbulent flow in a circular tube of diameter $D$, the viscous force per unit volume is; $F_{S}=$ $\frac{4 f\left(\frac{1}{2} \rho u^{2}\right)}{D}$, where the friction factor $f$ is given by [8]; $f=\frac{0.046}{(R e)^{0.2}}$. Substitute Eq. (1) into Eq. (2) with using the definitions of $u$ and $F_{S}$ and integrate around the loop, the momentum equation will be;

$$
\oint \frac{W^{2}}{A^{2}(s)} \frac{d v}{d s} d s=-\oint \frac{d P}{d s} d s-\oint \frac{4\left(\frac{1}{2} f W^{2}\right)}{\rho A^{2}(s) D(s)} d s-\oint \rho g \cos (\theta) d s
$$

where $v$ is the fluid specific volume and $P$ is the primary pressure. The pressure term of Eq. (4) will vanish. For strongly turbulent flow, it can be assumed that the friction coefficient is constant and equal to $\bar{f}$ for a given primary pressure. Noting that $A$ and $D$ are given by their average values over the loop and that $d z=d s \cos (\theta)$, Eq. (4) becomes;

$$
-g \oint \rho d z=\frac{\frac{1}{2}(4 \bar{f}) W^{2}}{\bar{A}^{2} \bar{D}} \oint \frac{d s}{\rho}
$$

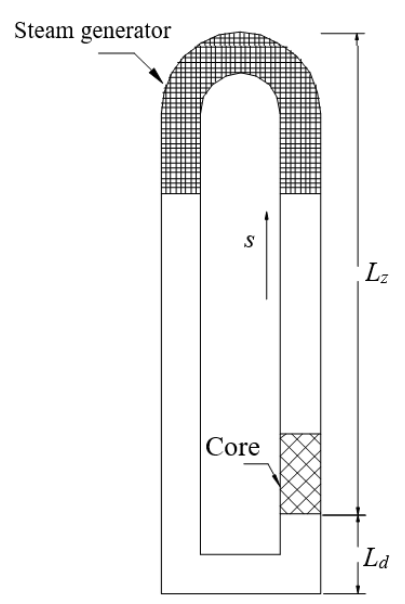

Fig. 1: One-dimensional loop. 
From Fig. 1;

$$
\oint \rho d z=\int_{0}^{L_{z}} \rho_{1} d z+\int_{L_{z}}^{0} \rho_{1} d z+\int_{0}^{-L_{d}} \rho_{2} d z+\int_{-L_{d}}^{0} \rho_{2} d z=\int_{0}^{L_{z}} \rho_{1} d z-\int_{0}^{L_{z}} \rho_{2} d z=\left(\bar{\rho}_{1}-\bar{\rho}_{2}\right) L_{z}
$$

Also, from Fig. 1;

$$
\oint \frac{d s}{\rho}=\oint v d s=\bar{v} L_{t}
$$

where $\rho_{1}$ and $\rho_{2}$ are the fluid densities of the system up-flow and down-flow sides respectively, $\bar{\rho}_{1}$ and $\bar{\rho}_{2}$ are the average values of the fluid densities $\rho_{1}$ and $\rho_{2}$ respectively, $\bar{v}$ is the average value of the fluid specific volume, $L_{z}$, and $L_{d}$ are the heights of the PWR and downcomer systems respectively, and $L_{t}$ is the total circulation length. Substitute Eqs. (6) and (7) into Eq. (5) and rearranging, to get;

$$
\frac{\frac{1}{2}(4 \bar{f}) W^{2}}{\bar{A}^{2} \bar{D}} \bar{v} L_{t}=g\left(\bar{\rho}_{2}-\bar{\rho}_{1}\right) L_{z}
$$

where $\bar{v}$ is the average fluid specific volume, and $L_{t}$ is the total circulation length. Equation (8) can be written as;

$$
W=\frac{\widehat{W}}{\bar{\rho}_{o}} \sqrt{\frac{\left(\bar{\rho}_{2}-\bar{\rho}_{1)}\right.}{\bar{v}} \frac{\bar{f}_{o}}{\bar{f}}}
$$

where; $\widehat{W}^{2}=\frac{2 g L_{z} \bar{D} \bar{\rho}_{o}^{2} \bar{A}^{2}}{4 \bar{f}_{o} L_{t}}$ which has the dimensions of flow rate and $\bar{f}_{o}$ and $\bar{\rho}_{o}$ are respectively the overall system average friction coefficient and the density corresponding to the conditions at the beginning of NC. The value of $\bar{\rho}_{1}$ is obtained from volume averaging of densities over the up-flow side of the PWR system elements. The energy Eq. (3) can be written separately for each component of the loop. The term $\left(-\frac{d q}{d s}\right)$ in Eq. (3) is equal to $\left(-\bar{U} \pi D_{s} n\left(T-T_{s e c}\right)\right)$ for the heat sink and zero for the (insulated) pipes. For the heated section, it depends upon the input power distribution. It is well known that the power distribution in a typical PWR core is far from being uniform. In this paper, to simplify derivations, uniform input power distribution is taken into consideration. For a uniformly distributed input power, the term $\left(-\frac{d q}{d s}\right)$ is equal to $\frac{Q_{o}}{L_{c}}$ for the heated section, where $Q_{o}$ is the heat input power, and $L_{c}$ is the length of flow channel in the core.

\subsection{Combined Single and Two-Phase Loop Flow}

Consider the loop to contain both single and two-phase flow, with unequal densities $\bar{\rho}_{1}$ and $\bar{\rho}_{2}$, on the hot and cold sides, respectively (Fig. 2). For the combined single and two-phase loop flow, the energy equation for single-phase regions can be written as; 


$$
C W \frac{d T}{d s}=\left\{\begin{array}{cc}
Q_{o} / L_{c} & \text { (Non Boiling height of heat source) } \\
-\bar{U}_{1} \pi D_{s} n\left(T_{s}-T_{s e c}\right) & \text { (Non Boiling length of steam generator) } \\
0 & \text { (Cold leg pipe) }
\end{array}\right.
$$

and for two-phase regions can be written as;

$$
W \frac{d h}{d s}=\left\{\begin{array}{cc}
Q_{o} / L_{c} & \text { (Boiling height of heat source) } \\
-\bar{U}_{2} \pi D_{s} n\left(T_{\text {sat }}-T_{\text {sec }}\right) & \text { (Boiling length of steam generator) } \\
0 & \text { (hot leg pipe) }
\end{array}\right.
$$

where; $C$ is the specific heat capacity that can be found from; $C=\frac{h_{f}-h_{i}}{T_{s a t}-T_{i}}, h$ is the specific enthalpy, $\bar{U}_{1}$ and $\bar{U}_{2}$ are the overall heat transfer coefficient for single and two-phase respectively, $D_{s}$ is the tube diameter of the steam generator, $n$ is the number of tubes in the steam generator, $T_{s}$ is the temperature of the steam generator, $T_{s a t}$ is the saturated temperature, and $T_{\text {sec }}$ is the temperature of the secondary side of the steam generator.

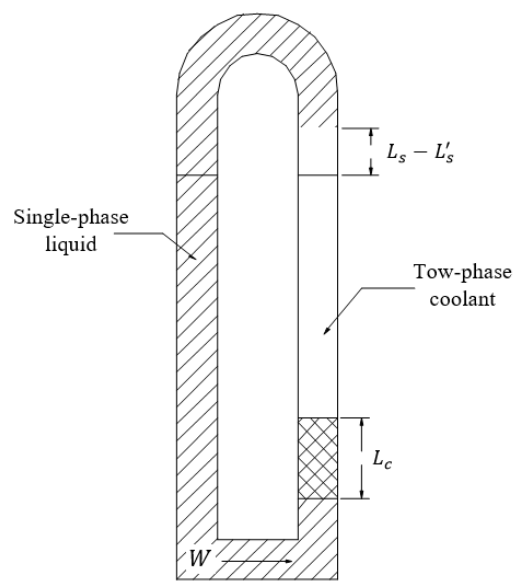

Fig. 2: Combined single and two-phase loop.

The solution of Eq. (10c) yields uniform temperature in the cold leg pipe, which implies that the steam generator outlet and core inlet temperatures are at cold leg temperature $T_{i}$. The solution of Eq. (11c) yields uniform enthalpy and hence quality in the hot leg pipe, which implies, that the outlet core and inlet steam generator qualities are at $x_{o}$. The exact solution of Eq. (10a) for a uniformly distributed input power is a linear temperature profile in the non-boiling height of the core $T_{c}$;

$$
T_{c}=T_{i}+\frac{Q_{o}}{C W} \frac{z}{L_{c}} \quad 0 \leq z \leq L_{c}^{\prime}
$$

The solution of Eq. (10b) is a temperature distribution of the non-boiling length of the primary side of the steam generator $T_{S}$

$$
T_{s}=T_{s e c}+\left(T_{s a t}-T_{s e c}\right) e^{-\frac{\bar{U}_{1} \pi D_{s} n}{C W}\left(z-L_{s}+L_{s}^{\prime}\right)} \quad\left(L_{s}-L_{s}^{\prime}\right) \leq z \leq L_{s}
$$


where $\bar{U}_{1}$ is the average overall heat transfer coefficient of the up-flow side, and $z$, is measured from the bottom of the primary side of the hot leg of the steam generator. The solution of Eq. (11a) yields the quality distribution of the boiling height of the core. Utilizing the definition of the specific enthalpy, $h=h_{f}+x h_{f g}$ in Eq. (11a) and integrating, yields;

$$
x_{c}=\frac{Q_{o}}{W h_{f g}} \frac{z-L_{c}^{\prime}}{L_{c}} \quad\left(L_{c}^{\prime} \leq z \leq L_{c}\right)
$$

where $h_{f}$ is the saturated liquid specific enthalpy and $h_{f g}$ is the latent heat of vaporization. Setting $z=L_{C}$ in the above equation, yields the quality at the upper plenum, $x_{o}$;

$$
x_{o}=\frac{Q_{o}}{W h_{f g}} \frac{L_{c}-L_{c}^{\prime}}{L_{c}}=\frac{Q_{o}}{W h_{f g}}\left(1-L_{c}^{*}\right)
$$

The solution of Eq. (11b) yields the quality distribution along the boiling length of the steam generator;

$$
x_{s}=x_{o}-\frac{\bar{U}_{2} \pi D_{s} n\left(T_{s a t}-T_{s e c}\right)}{W h_{f g}} z \quad\left(0 \leq z \leq L_{s}-L_{s}^{\prime}\right)
$$

The temperature and quality profiles described by Eqs. (12) through (16) are used to obtain the density distribution for each elemnet of a PWR loop. For incompressible single-phase flow, it will be assumed that the Boussinesq approximations for density are valid. Thus; $\rho=\rho_{l}\left[1-\beta\left(T-T_{l}\right)\right]$. This equation might be regarded as a Taylor-mode equation of state and can be written as; $\rho=a+b T$. The constants $a$ and $b$ can be calculated from; $a=\rho_{l}\left(1+\beta T_{l}\right)$ and $b=-\rho_{l} \beta$, where $\rho_{l}$ and $\beta$ are evaluated at some reference temperature $T_{l}$, and $\beta$, the coefficient of volume expansion, is defined by; $\beta=\frac{1}{v}\left(\frac{\partial v}{\partial T}\right)_{P}$. The average density for each element can be derived as follows:

For the core:

$$
\bar{\rho}_{c}=\bar{\rho}_{c 1 \emptyset}+\bar{\rho}_{c 2 \emptyset}
$$

where; $\bar{\rho}_{c 1 \varnothing}$ and $\bar{\rho}_{c 2 \varnothing}$ are the average core densities for single-phase and two-phase respectively.

$$
\begin{array}{ll}
\text { For the hot leg and upper plenum: } & \bar{\rho}_{u}=\frac{1}{v_{f}+v_{f g} x_{o}} \\
\text { For the steam generator: } & \bar{\rho}_{s}=\bar{\rho}_{s 2 \phi}+\bar{\rho}_{s 1 \phi}
\end{array}
$$

$$
\begin{gathered}
\bar{\rho}_{c 1 \varnothing}=\frac{\int_{0}^{L_{c}^{\prime}} \rho_{c 1 \emptyset} d z}{L_{c}}=L_{c}^{*}\left[a+b T_{i}+\frac{b Q_{o}}{2 C W} L_{c}^{*}\right] \\
\bar{\rho}_{c 2 \varnothing}=\frac{\int_{L_{c}^{\prime}}^{L_{c}} \rho_{c 2 \varnothing} d z}{L_{c}}=\frac{W h_{f g}}{v_{f g} Q_{o}} \ln \left[\frac{v_{f}+v_{f g} x_{o}}{v_{f}}\right] \\
\bar{\rho}_{u}=\frac{1}{v_{f}+v_{f g} x_{o}}
\end{gathered}
$$

where; $v_{f}$ is the saturated liquid specific volume, $\bar{\rho}_{s 2 \phi}$ and $\bar{\rho}_{s 1 \phi}$ are the average steam generator densities for two-phase and single-phase respectively. 


$$
\begin{gathered}
\bar{\rho}_{s 2 \phi}=\frac{\int_{0}^{L_{s}-L_{s}^{\prime}} \rho_{s 2 \phi} d z}{\frac{L_{s}}{2}}=\frac{2 W h_{f g}}{v_{f g} \bar{U}_{2} \pi D_{s} n\left(T_{s a t}-T_{s e c}\right) L_{s}} \ln \left[\frac{v_{f}+v_{f g} x_{o}}{\left.v_{f}+v_{f g} x_{o}-\frac{v_{f g} \bar{U}_{2} \pi D_{s} n\left(T_{s a t}-T_{s e c}\right)\left(L_{s}-L_{s}^{\prime}\right)}{W h_{f g}}\right]}\right. \\
\bar{\rho}_{s 1 \phi}=\frac{\int_{L_{s}-L_{s}^{\prime}}^{L_{s} / 2} \rho_{s 1 \phi} d z}{\frac{L_{s}}{2}}=\left(2 L_{S}^{*}-1\right)\left[a+b T_{s e c}-\frac{b\left(T_{s a t}-T_{s e c}\right) C W}{\bar{U}_{1} \pi D_{s} n\left(L_{s}^{\prime}-\frac{L_{s}}{2}\right)}\right]\left\{e^{-\frac{\bar{U}_{1} \pi D_{s} n}{C W}\left(L_{s}^{\prime}-\frac{L_{s}}{2}\right)}-1\right\} \\
\bar{\rho}_{2}=a+b\left[T_{\text {sec }}+\left(T_{\text {sat }}-T_{\text {sec }}\right) e^{-\frac{\bar{U}_{1} \pi D_{s} n}{C W}\left(L_{s}^{\prime}-\frac{L_{s}}{2}\right)}\right]
\end{gathered}
$$

where $\bar{\rho}_{2}$ is the average density of the down-flow side. The term $\bar{v}$, in Eq. (9), can be obtained from volume averaging over the PWR system elements.

$$
\begin{aligned}
& \bar{v}_{c}=\bar{v}_{c 1 \phi}+\bar{v}_{c 2 \phi}=\frac{\int_{0}^{L_{c}^{\prime}} \frac{d z}{\rho_{c 1 \phi}}+\int_{L_{c}^{\prime}}^{L_{c}} \frac{d z}{\rho_{c 2 \phi}}}{L_{c}}=\left(1-L_{c}^{*}\right)\left[v_{f}+\frac{1}{2} v_{f g} x_{o}\right]+L_{c}^{*} v_{1} \\
& \bar{v}_{u}=v_{f}+v_{f g} x_{o} \\
& \bar{v}_{s}=\bar{v}_{s 2 \phi}+\bar{v}_{s 1 \phi}=\frac{\int_{0}^{L_{s}-L_{s}^{\prime}} \frac{d z}{\rho_{s 2 \phi}}+\int_{L_{s}-L_{s}^{\prime}}^{L_{s} / 2} \frac{d z}{\rho_{s 1 \phi}}}{L_{s} / 2} \\
& =2\left(1-L_{s}^{*}\right)\left[v_{f}+v_{f g} x_{o}-\frac{v_{f g} \bar{U}_{2} \pi D_{s} n\left(T_{s a t}-T_{s e c}\right.}{2 W h_{f g}}\left(L_{s}-L_{s}^{\prime}\right)\right]+L_{s}^{*} v_{1} \\
& \bar{v}_{2}=v_{1}
\end{aligned}
$$

where, $\bar{v}_{c}, \bar{v}_{s}, \bar{v}_{2}$, and $\bar{v}_{1}$ are the specific volumes of the core, steam generator, down-flow, and up-flow side respectively. The relations (25) through (28) can now be used to calculate $\bar{v}$ and also the mass flow rate (Eq. (9)) after evaluating $\bar{\rho}_{1}$ and $\bar{\rho}_{2}$. The inlet temperature of the core, $T_{i}$, is obtained from Eq. (13) by setting $z=L_{s} / 2$, thus;

$$
T_{i}=T_{s e c}+\left(T_{s a t}-T_{s e c}\right) e^{-\frac{\bar{U}_{1} \pi D_{s} n}{C W}\left(L_{S}^{\prime}-\frac{L_{s}}{2}\right)}
$$

The outlet temperature of the core $\left(T_{o}\right)$ is equal to the saturation temperature at the primary loop pressure $\left(T_{\text {sat }}\right)$. Hence the temperature difference of the core will be;

$$
\Delta T_{\text {core }}=T_{o}-T_{i}=\left(T_{\text {sat }}-T_{\text {sec }}\right)\left(1-e^{-\frac{\bar{U}_{1} \pi D_{S} n}{C W}\left(L_{S}^{\prime}-\frac{L_{S}}{2}\right)}\right)
$$

In order to evaluate correctly the driving head in a natural circulation reactor, it is necessary to calculate the non-boiling heights in the channels of the core and steam generator and the average overall heat transfer coefficient of single and twophase flow in the channels of steam generator. The non-boiling height of the core $L_{c}^{\prime}$, is defined as the height where the coolant becomes saturated. The remainder of the channel is that in which boiling takes place and is called the boiling height, $L_{c}-L_{c}^{\prime}$. Some sub-cooled boiling occur in $L_{c}^{\prime}$ but will have a little effect on density. The ratio, $L_{c}^{\prime} / L_{c}$, can be evaluated from the ratio of sensible heat $\left(q_{s}\right)$ added to total heat $\left(Q_{0}\right)$ added in the channel [9]. 


$$
\frac{q_{s}}{Q_{0}}=\frac{W\left(h_{f}-h_{i}\right)}{Q_{0}}=\frac{L_{c}^{\prime}}{L_{c}}=L_{c}^{*}
$$

Similarly, for the primary side of the steam generator, the non-boiling length is $L_{s}^{\prime}$, and the boiling length is $L_{s}-L_{s}^{\prime}$. $L_{s}^{\prime}$, can be evaluated by setting $x_{s}=0$ at $z=L_{s}-L_{S}^{\prime}$, thus;

$$
L_{s}^{\prime}=L_{s}-\frac{x_{o} W h_{f g}}{\bar{U}_{2} \pi D_{s} n\left(T_{s a t}-T_{s e c}\right)}
$$

\subsection{Overall Heat Transfer Coefficient}

The overall heat transfer coefficient for the radial flow of heat across a circular tube based on the internal tube surface is [9];

$$
\frac{1}{\overline{\bar{U}}_{i}}=\frac{1}{H_{i}}+\frac{A_{i} t_{w}}{A_{m w} K_{w}}+\frac{A_{i}}{A_{o} H_{o}}
$$

where, $H_{i}$ and $H_{o}$ are the heat transfer coefficient inside and outside the tube respectively, $A_{i}$ and $A_{o}$ are the internal and external tube surface areas respectively, $A_{m w}$ is the mean wall area for heat transfer, $t_{w}$ is the wall thickness, and $K_{w}$ is the thermal conductivity of the tube wall. The heat transfer resistance by conduction can be neglected because of small thickness of steam generator tubes. For single- and two-phase flow in the steam generator, convective heat transfer on the inside of the tubes and nucleate boiling on the secondary side of the tubes will occur [10]. The heat transfer coefficients in the primary side of steam generator $H_{i}$, have been taken, from forced flow correlations. From the above considerations, the average overall heat transfer coefficient for single-phase flow, $\bar{U}_{1 \phi}$, and two-phase flow, $\bar{U}_{2 \phi}$, can be written as;

$$
\begin{aligned}
& \bar{U}_{1 \phi}=\frac{1}{H_{i 1 \phi}}+\frac{A_{i}}{A_{o} H_{o 1 \phi}} \\
& \bar{U}_{2 \phi}=\frac{1}{H_{i 2 \phi}}+\frac{A_{i}}{A_{o} H_{o 2 \phi}}
\end{aligned}
$$

where; $H_{i 1 \phi}$ is the forced convection heat transfere coefficient, for single-phase, on the primary side of steam generator, which can be evaluated from the following correlation [8]:

$$
H_{i 1 \phi}=0.023 \operatorname{Re}^{0.8} \operatorname{Pr}^{0.3} \frac{k}{d_{e}},
$$

and; $H_{i 2 \phi}$ is the heat transfer coefficient for two-phase in the primary side of steam generator for bubble flow, which can be taken from the following correlation [11]:

$$
H_{i 1 \phi}=0.023 \operatorname{Re}^{0.8} \operatorname{Pr}^{0.3} \frac{k}{d_{e}},
$$


where Re is the Reynolds number, $\operatorname{Pr}$ is the Prandtle number, $k$ is the fluid thermal conductivity, and $d_{e}$ is the equivalent diameter of flow channel. $H_{o 1 \phi}$ and $H_{o 2 \phi}$ are the heat transfer coefficients for nucleate boiling of the secondary side of steam generator, which can be taken from the following correlations [11]:

$$
\begin{gathered}
H_{o 1 \phi}=2.5551 \times 10^{-3} e^{0.6446133 P_{s e c}}\left(T_{\text {bulk }}-T_{\text {sec }}\right)^{3}, \\
H_{o 2 \phi}=2.5551 \times 10^{-3} e^{0.6446133 P_{\text {sec }}}\left(T_{\text {sat }}-T_{\text {sec }}\right)^{3}
\end{gathered}
$$

where $P_{s e c}$ is the pressure in the secondary side of steam generator in $\mathrm{MPa}, T$ is the temperature in ${ }^{\circ} \mathrm{C}$ and $H_{\mathrm{o}}$ is the heat transfer coefficient in $\frac{\mathrm{kW}}{\mathrm{m}^{2} .{ }^{\circ} \mathrm{C}}$. To evaluate the two phase Reynolds number, two phase dynamic viscosity, $\mu_{2 \varnothing}$, must be determined from the following equation:

$$
\frac{1}{\mu_{2 \phi}}=\frac{\bar{x}_{s}}{\mu_{g}}+\frac{1-\bar{x}_{s}}{\mu_{f}}
$$

where; $\bar{x}_{s}$ is the average quality of stream generator which is equal to $\frac{x_{o}}{2}$ (for the combined single and two phase flow) and $\frac{x_{i}+x_{o}}{2}$ (for the two phase flow), and $\mu_{g}$ and $\mu_{f}$ are the dynamic viscosities of saturated vapor and saturated liquid respectively.

\section{Results}

In this paper, in order to obtain the results of the NC parameters, iterations are used. The values of $\rho_{o}$ and $f_{o}$ for every reactor power $Q_{o}$, can be determined when the primary pressure is at its initial value $\left(P_{o}=11.2 \mathrm{MPa}\right)$ (see Table 1$)$. The results of this paper are compared with the experimental data from the Semiscale Mod-2A system for three different powers (30, 60 and $100 \mathrm{KW}$ ) [10]. The date required for the calculations in this paper that concern the Semiscale Mod-2A system are shown in Table $2[10,12,13]$. Figures 3 to 6 show the calculated parameters of the natural circulation as a function of the primary pressure as well as the comparisons of these data with the experimental results of Semiscale Mod-2A system.

Table 1: Values of $\bar{\rho}_{o}$ and $\bar{f}_{o}$ as a function of core power.

\begin{tabular}{|c|c|c|c|}
\hline $\boldsymbol{Q}_{\boldsymbol{o}}(\mathbf{K W})$ & 30 & 60 & 100 \\
\hline$\overline{\boldsymbol{\rho}}_{\boldsymbol{o}}$ & 743.2258 & 734.6119 & 730.6987 \\
\hline$\overline{\boldsymbol{f}}_{\boldsymbol{o}}$ & $4.847 \times 10^{-3}$ & $4.614 \times 10^{-3}$ & $4.462 \times 10^{-3}$ \\
\hline
\end{tabular}

Table 2: Summary of the design and operating data for the Semiscale model.

\begin{tabular}{|c|c|c|c|c|c|}
\hline Parameter & Data & Parameter & Data & Parameter & Data \\
\hline$A_{i}$ & $3.05 \times 10^{-4} \mathrm{~m}^{2}$ & $L_{\mathrm{s}}$ & $20 \mathrm{~m}$ & $P_{\mathrm{sec}}$ & $6 \mathrm{MPa}$ \\
\hline$A_{o}$ & $3.87 \times 10^{-4} \mathrm{~m}^{2}$ & $V_{\mathrm{c}}$ & 0.06 & $T_{\mathrm{sec}}$ & $275.64^{\circ} \mathrm{C}$ \\
\hline$D_{\mathrm{s}}$ & $0.0222 \mathrm{~m}$ & $V_{\mathrm{u}}$ & 0.18 & $T_{1}$ & $T_{\text {bulk }}$ \\
\hline$t_{w}$ & $0.00124 \mathrm{~m}$ & $V_{\mathrm{s}}$ & 0.22 & $\rho_{1}$ & $\rho_{\mathrm{bulk}}$ \\
\hline$n$ & 6 & $V_{\mathrm{cl}}$ & 0.29 & $\widehat{W}$ & $1.35 \mathrm{Kg} / \mathrm{sec}$ \\
\hline$L_{\mathrm{c}}$ & $3.66 \mathrm{~m}$ & full core power (scaled) & $2 \mathrm{MW}$ & & \\
\hline
\end{tabular}




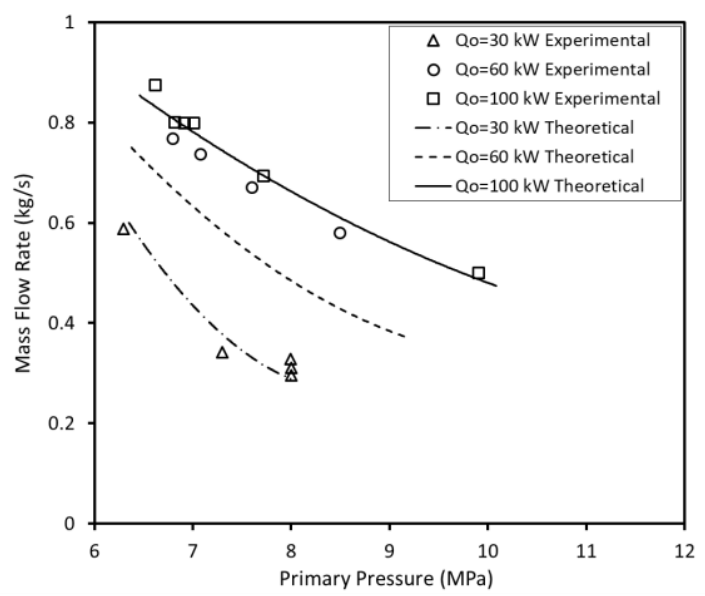

Fig. 3: Comparison of theory with Semiscale PWR data of Mass flow rate versus Primary pressure for different core powers.

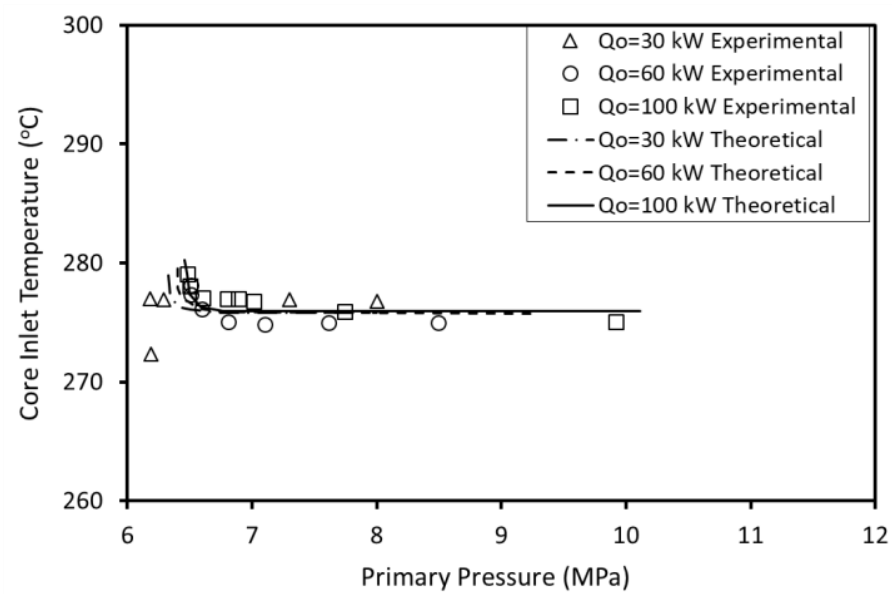

Fig. 4: Comparison of theory with Semiscale PWR data of Core inlet temperature versus Primary pressure for different core powers.

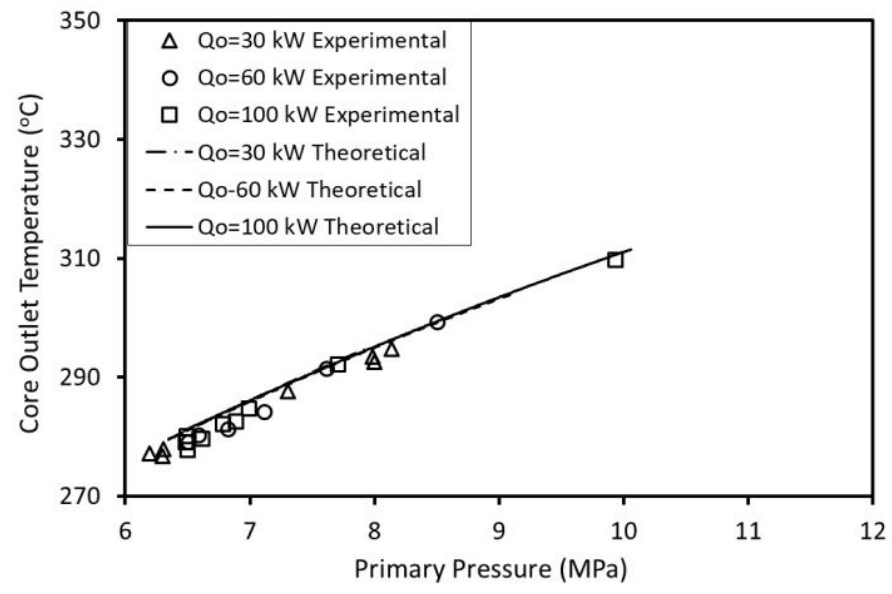

Fig. 5: Comparison of theory with Semiscale PWR data of Core outlet temperature versus Primary pressure for different core powers. 


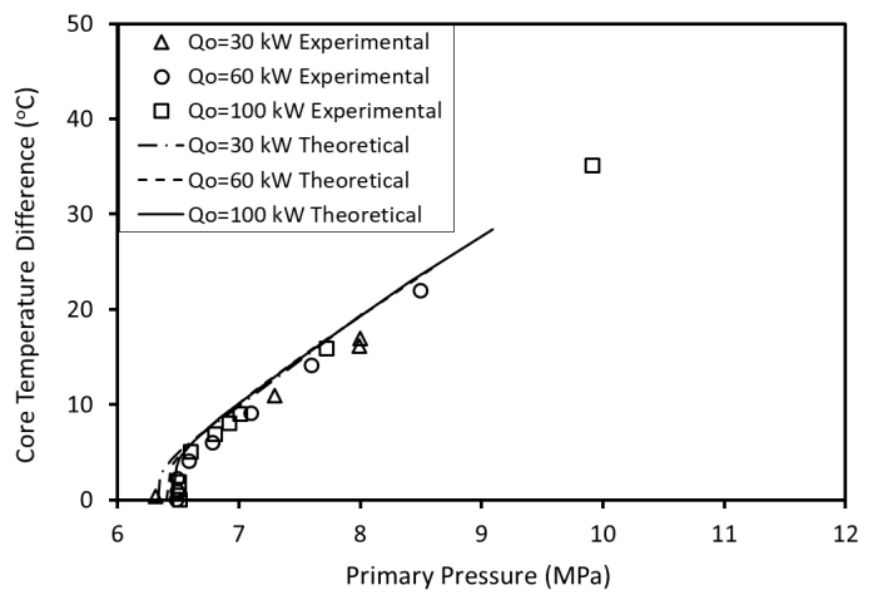

Fig. 6: Comparison of theory with Semiscale PWR data of Core temperature difference versus Primary pressure for different core powers.

Natural circulation starts at an initial primary pressure of 11.2 MPa where only single-phase flow exists. As the draining is progressed in the primary loop of the PWR, the fluid level in the upper plenum will continue to fall down until it reaches the hot leg whereby, combined single and two-phase flow in the natural circulation will commence, and $0.5 \leq L_{\mathrm{s}}^{*} \leq 1$. From Figs. 3 to 6 , it can be noted that the occurrence of combined single and two-phase natural circulation, takes place over a broad band of system pressures. In Fig. 3, the mass flow rate is observed to increase gradually as the primary pressure continue to fall until the maximum value is eventually reached. This increase in the mass flow rate is attributed to the sharp decrease of the two-phase fluid density in the core, hot leg, and up-flow side of the steam generator with no corresponding decrease in the single-phase density of the down-flow side of the steam generator and exit piping. Figure 4 shows the core inlet temperature as a function of primary pressure. In this figure, initially the cold leg temperature is seen to be insensitive to reduction in primary pressure, but finally it increases until it reaches the saturation temperature at the maximum mass flow rate. This is due to the fact that the overall heat transfer coefficient is sufficient to condense all of the voids entering the steam generator within its up-flow side. Therefore, the fluid temperature and density in the down-flow side of the tubes remain nearly constant, while the average density of the up-flow side of the steam generator decreases, as the pressure is decreased. Hence the overall driving head will increase with decreasing pressure causing an increase in the mass flow rate.

Figure 5 shows the core outlet temperature (hot leg temperature) as a function of a primary pressure. It can be seen that the hot leg temperature is maintained at saturation, so that it decreases as the primary pressure is decreased. In Fig. 6, the core differential temperature is seen to decrease gradually with decreasing primary pressure and this is due to a combination of decreasing hot leg temperature and increasing mass flow rate. Figures 3 to 7 also show the effect of the reactor power on the NC parameters. In general, it can be seen that the overall trends for the three power levels are similar. The primary pressure, at the starting of the combined single and two-phase NC, is greater the higher the core power, while at the ending point is approximately the same. In Fig.3, the loop mass flow rate is observed to increase with increasing core power. This behaviour can be explained as due to the increase in the overall density gradient between the hot and cold sides of the systems, resulting in higher loop mass flow rates. Figures 4 to 6 reveal that the cold and hot leg temperatures as well as the core temperature difference are practically independent of core power.

It is noted that the present theory is capable in predicting the correct trend over the whole range of primary pressure for combined single and two-phase flow NC and show reasonable agreement with the experimental results. Table 3 shows the maximum error between the experimental and theoretical results of $\mathrm{NC}$ parameters. The discrepancies in the results can be attributed to different reasons. The first one is the uncertainties in experimental data, which were estimated [12] as \pm 0.033 $\mathrm{Kg} / \mathrm{s}$ for mass flow rate, $\pm 5^{\circ} \mathrm{C}$ for core temperature difference, and $\pm 0.4 \mathrm{MPa}$ for secondary pressure. The second reason is the model approximations. The one-dimensional model used is inadequate, under certain operating conditions and in regions where abrupt changes in flow area occurs, to describe the three-dimensional nature of the flow. Another reason is 
the usage of forced flow correlations for heat transfer coefficient and friction factor in NC flow and neglecting the effect of form losses in the various components in the loop.

Table 3: Maximum absolute errors between experimental and theoretical results for the NC parameters versus the primary pressure for different power values.

\begin{tabular}{|c|c|c|c|c|}
\cline { 2 - 5 } \multicolumn{1}{c|}{} & \multicolumn{4}{c|}{ Max. error (\%) } \\
\hline Power (KW) & $\boldsymbol{W}$ & $\boldsymbol{T}_{\boldsymbol{i}}$ & $\boldsymbol{T}_{\boldsymbol{o}}$ & $\Delta \boldsymbol{T}_{\text {core }}$ \\
\hline 30 & 11.31 & 0.75 & 8.15 & 7 \\
\hline 60 & 26.17 & 0.73 & 2.72 & 20.25 \\
\hline 100 & 4.95 & 0.43 & 2.62 & 25.3 \\
\hline
\end{tabular}

\section{Conclusion}

In this paper, one dimensional continuity, momentum and energy equations are solved to find the parameters of the NC flow versus the primary pressure in a PWR plant. It is found that the NC in a PWR can play an important role in rejecting the core decay heat to the secondary side of the steam generator over a wide range of a primary coolant pressure, and a core decay power range of 1.5 to $5 \%$ of full power. Also, it is found that as the primary pressure decreases, the core differential temperature decreases while the loop mass flow rate increases. This paper demonstrated that the combined single and twophase NC flow rate in PWRs are strongly dependent on primary loop pressure, and weakly dependent on power level.

\section{References}

[1] Y.Zvirin, "A review of natural circulation loops in pressurized water reactors and other systems,". Nuclear Engineering and Design, vol. 67, pp. 203-225, 1981.

[2] L. W. Ward, "Simplified small-break blowdown models," Trans. Am. Nucl. Soc., vol. 18, pp. 232, 1974.

[3] D. J. Shimeck and G. W. Johnsen, "Natural circulation cooling in a pressurized water reactor geometry under accidentinduced conditions". Nucl. Sci. Engrg., vol. 88, pp. 311-320, 1984.

[4] R. T. Fernandez, J. P. Sursock and R. L. Kiang, "Reflux boiling heat removal in a scaled TMI-2 system test facility," in proceedings of the ANS / ENS Thermal Reactor Safety Meeting, Knoxville, Tenn, April, 1980.

[5] M. W. Abdulrahman, "Heat transfer in a tubular reforming catalyst bed: Analytical modelling," in proceedings of the 6th International Conference of Fluid Flow, Heat and Mass Transfer (FFHMT'19), 2019.

[6] M. W. Abdulrahman, "Exact analytical solution for two-dimensional heat transfer equation through a packed bed reactor," in Proceedings of the $7^{\text {th }}$ World Congress on Mechanical, Chemical, and Material Engineering (MCM'20), 2020.

[7] J. B. Keller, "Oscillations in a model of thermal convection,” J. Fluid Mech., vol. 26, pp. 599-606, 1966.

[8] A. Shitzer, D. Kalmanoviz, Y. Zvirin and G. Grossman, "Experiments with a flat-plate solar water heating system in thermosyphonic flow," Solar Energy, vol. 22, pp. 27-35, 1979.

[9] M. M. El-Wakil, Nuclear power engineering. Mc Graw-Hill Book Company, 1962.

[10] G. G. Loomis and K. Soda, "Results of the Semiscale Mod-2A natural circulation experiments," NRC Report, Idaho National Engineering Lab., NUREG / CR-2335, September, 1982.

[11] Nicholas P. Cheremisinoff, Heat transfer pocket handbook. 1984.

[12]D. J. Shimeck and G. W. Johnsen, "Natural circulation cooling in a pressurized water reactor geometry aunder accident-induced conditions," Nucl. Sci. Eng., vol. 88, pp. 311-320, 1984.

[13] R. B. Duffey and J. P. Sursock, "Natural circulation phenomena to small breaks and transients," Nuclear Engineering and Design, vol. 102, pp. 115-128, 1987. 